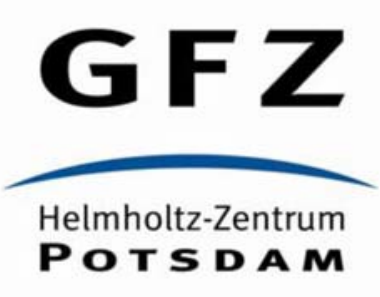

Originally published as:

Wendi, D., Marwan, N. (2018): Extended recurrence plot and quantification for noisy continuous dynamical systems. - Chaos, 28, 8, 085722.

DOI: http://doi.org/10.1063/1.5025485 


\title{
Extended recurrence plot and quantification for noisy continuous dynamical systems
}

\author{
Dadiyorto Wendi ${ }^{1,2,3}$ and Norbert Marwan ${ }^{2}$ \\ ${ }^{1}$ Institute of Earth and Environmental Science, University of Potsdam, Potsdam-Golm 14476, Germany \\ ${ }^{2}$ Potsdam Institute for Climate Impact Research (PIK), Potsdam 14412, Germany \\ ${ }^{3}$ Section 5.4 Hydrology, GFZ German Research Centre for Geosciences, Potsdam 14473, Germany
}

(Received 9 February 2018; accepted 2 May 2018; published online 30 August 2018)

\begin{abstract}
One main challenge in constructing a reliable recurrence plot (RP) and, hence, its quantification [recurrence quantification analysis (RQA)] of a continuous dynamical system is the induced noise that is commonly found in observation time series. This induced noise is known to cause disrupted and deviated diagonal lines despite the known deterministic features and, hence, biases the diagonal line based RQA measures and can lead to misleading conclusions. Although discontinuous lines can be further connected by increasing the recurrence threshold, such an approach triggers thick lines in the plot. However, thick lines also influence the RQA measures by artificially increasing the number of diagonals and the length of vertical lines [e.g., Determinism (DET) and Laminarity (LAM) become artificially higher]. To take on this challenge, an extended RQA approach for accounting disrupted and deviated diagonal lines is proposed. The approach uses the concept of a sliding diagonal window with minimal window size that tolerates the mentioned deviated lines and also considers a specified minimal lag between points as connected. This is meant to derive a similar determinism indicator for noisy signal where conventional RQA fails to capture. Additionally, an extended local minima approach to construct RP is also proposed to further reduce artificial block structures and vertical lines that potentially increase the associated RQA like LAM. The methodology and applicability of the extended local minima approach and DET equivalent measure are presented and discussed, respectively. Published by AIP Publishing. https://doi.org/10.1063/1.5025485
\end{abstract}

One main challenge in constructing a reliable recurrence plot (RP) and, hence, its quantification (RQA) of a continuous dynamical system is the induced noise that is commonly found in observation time series. This induced noise is known to cause disrupted and deviated diagonal lines despite the known deterministic features and, hence, biases the diagonal line based RQA. Therefore, an extended RQA approach using the sliding diagonal window for accounting disrupted and deviated diagonal lines is proposed. In addition, an extended local minima RP is also introduced to prevent RP's artifacts of noise induced signals caused by block structures and thick lines.

\section{INTRODUCTION}

As a fundamental property of many dynamical systems, recurrence can be exploited to characterize the system's behavior from their phase space topology. A recurrence plot (RP) is a visualization tool for the analysis of this property. In this study, the phase space reconstruction method of time delay embedding ${ }^{1,2}$ is used to create the topology of the system dynamics $\vec{x}_{i}$ from a variable $u_{i}$ [Eq. (1)]:

$$
\vec{x}_{i}=\sum_{i=1}^{m} u_{i+(j-i) \tau} \vec{e}_{j}
$$

where $i$ is the current time point and $j$ is other time point, $m$ is the embedding dimension, and $\tau$ is the time delay. The vectors $\left(\vec{e}_{j}\right)$ are unit vectors and span an orthogonal coordinate system $\left(\vec{e}_{i} \cdot \vec{e}_{j}\right)=\delta_{i, j}$.

The RP is basically the visual representation of the square matrix, in which the matrix elements correspond to those times at which a state of a dynamical system recurs (columns and rows correspond then to a certain pair of times). The calculation of a recurrence point as an element of the RP matrix $\mathbf{R}$ is usually based on Eq. (2). RPs are especially useful for analyzing non-stationary time series ${ }^{3,4}$

$$
R_{i, j}(\varepsilon)=\Theta\left(\varepsilon-\left\|\vec{x}_{i}-\vec{x}_{j}\right\|\right), \quad i, j=1, \ldots, N,
$$

where $N$ is the number of measured points $\vec{x}_{i}, \varepsilon$ is a threshold distance, $\|\cdot\|$ is a norm, and $\Theta(\cdot)$ is the Heaviside step function.

Besides using RP for the visual analysis of time series, $\mathrm{RP}$ can also be used quantitatively to unveil hidden structures from the series through recurrence quantification analysis (RQA). ${ }^{4,5}$ In RQA, the important elements are the diagonal and vertical lines because they reveal typical dynamical features of the investigated system, such as range of predictability, chaos-order, and chaos-chaos transition. ${ }^{6}$ One of the prominent diagonal line measures is determinism (DET), from which the system's predictability can be inferred:

$$
D E T=\frac{\sum_{d=d_{\min }}^{N} d P(d)}{\sum_{i, j}^{N} R_{i, j}},
$$

where $P(d)=\left\{d_{i} ; i=1, \ldots, N_{\mathrm{d}}\right\}$ is the histogram of the lengths $d$ of connected diagonal lines, and $N_{\mathrm{d}}$ is the absolute number of those diagonal lines. 
In addition, laminarity ( $L A M)$ is a measure based on the distribution of the RP's vertical lines:

$$
L A M=\frac{\sum_{v=v_{\min }}^{N} v P(v)}{\sum_{i, j}^{N} R_{i, j}},
$$

where $P(v)=\left\{v_{i} ; i=1, \ldots, N_{\mathrm{v}}\right\}$ is the histogram of the lengths $v$ of connected vertical structures and $N_{\mathrm{v}}$ is the absolute number of those vertical lines.

For a deterministic continuous dynamical system (including chaos), many diagonal lines in the RP are typical, leading to a high value of $D E T .^{7}$ However, single, isolated recurrence points can occur if states are rare, if they do not persist, or if they fluctuate heavily. For instance, stochastic or random signals would comprise such single points and result in a very low DET.

Nevertheless, pitfalls and artifacts are not unusual when constructing RP such as those occurring with the wrong choice of embedding parameters or high threshold $(\varepsilon) .^{7-9}$ Thick lines, for instance, could easily occur in continuous dynamical systems due to the temporal correlation of the phase space trajectory, and, hence causing the RP to contain redundant information. ${ }^{8}$ Such thick lines artificially increase the number of diagonal lines and even introduce vertical lines that lead to artifacts in RQA such as an increase in DET and $L A M$. With regard to these thick lines, Schultz et al. suggested a local minima based RP with additional threshold $\varepsilon$ to overcome this issue and demonstrated the benefits on the Lorenz system as an example. The minima are found in each column of the RP and should correspond to the closest neighbors of a state (within the range $\varepsilon$ ). Such a method is shown to minimize line thickness without requiring much computational effort as would be necessary for perpendicular or iso-directional RPs that had also been suggested to overcome the line thickness problem. Additionally, Schultz et al. concluded that such local minima based RP resulted in less dependency when choosing the $\varepsilon$. In our study, we call this approach LMT (i.e., local minima with threshold), while we refer the local minima without any constraint parameter as LM. LM and LMT methods' difference is further illustrated in Fig. 1(d), such that LM considers all local minima found, while LMT only considers the local minima below the $\varepsilon$, indicated by the region of orange shade.

However, in real world application, observation signals are often induced by noise, and that can lead to further artifacts in our RP and RQA. Especially, in the regular RP (using a distance threshold $\varepsilon$ ), the induced noise could easily disrupt and deviate the position of recurring points (Fig. 1). Nevertheless, such is no exception when using the LM approach, the mentioned artifacts are still present due to the induced noise. It is also important to note that in this study, we only consider the contamination of noise by adding Gaussian to a one dimensional input signal (i.e., of a continuous dynamical systems or in this case Lorenz) given the desired signal SNR (signal to noise ratio) in decibels.

The impact of noise is obvious in the traditional distance threshold based RP, LM, and LMT (Fig. 2). Visible block clusters can be observed in the threshold based RP

(a)

(b)

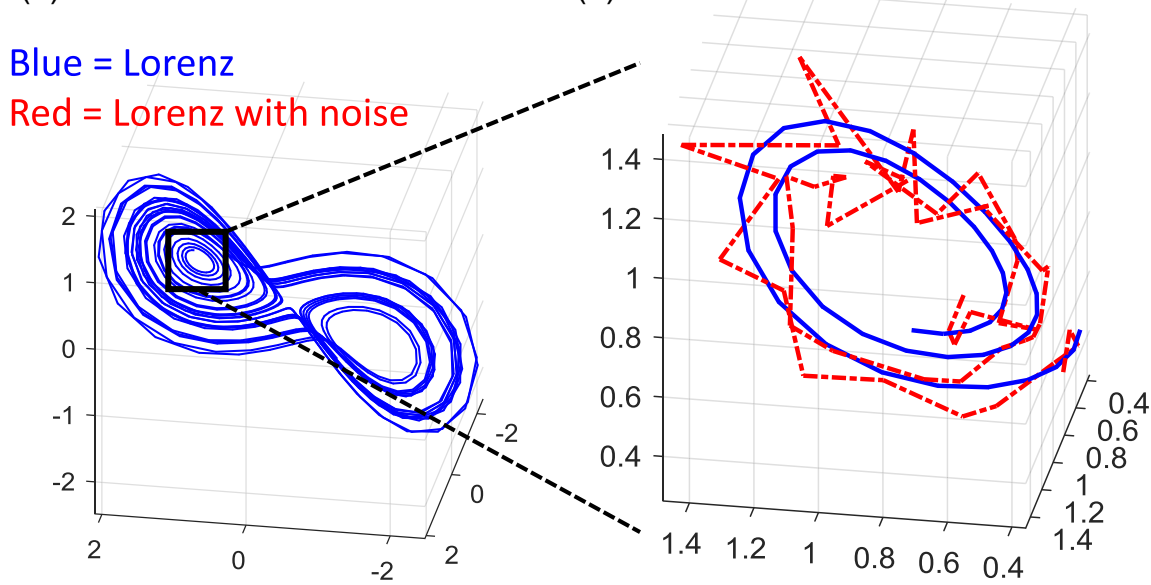

(c)

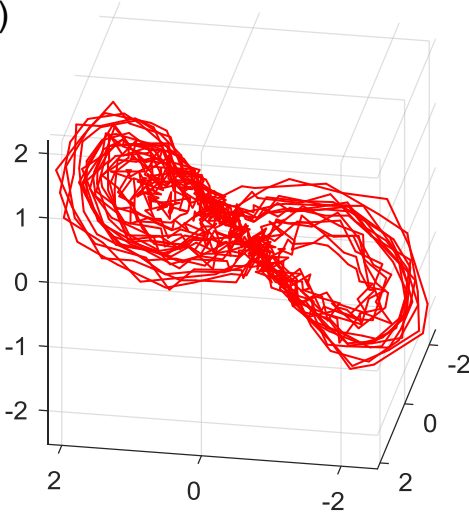

(d)

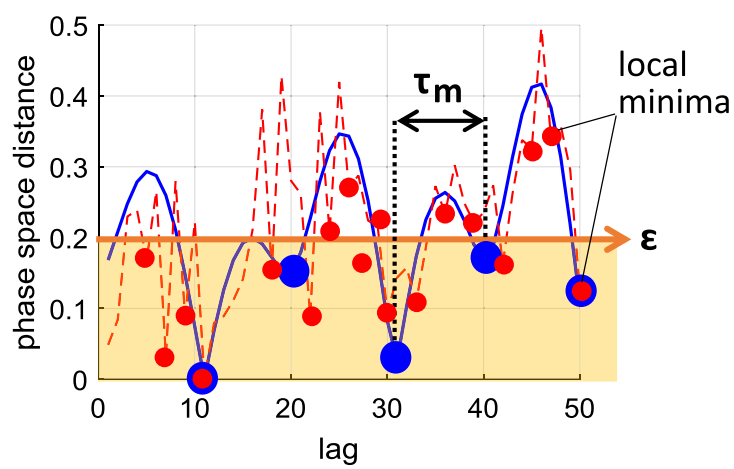

FIG. 1. Phase space trajectories of the Lorenz series ( $\frac{d x}{d t}$, with $\sigma=10$, $\rho=28, \beta=\frac{8}{3}$ ): (a) without noise and (b) with noise (SNR $=20 \mathrm{~dB}$ ); (c) zoomin view of both signal trajectories; and (d) extracted phase space distance (yaxis) between a selected point at the phase space trajectory and all other trajectory points by time lag (x-axis) and corresponding local minima (blue and red points, indicating local minima of no noise and noisy Lorenz, respectively). The shaded orange region refers to the region where local minima with threshold $(\varepsilon)$ are used for RP with the LMT approach. The embedding parameters of all phase space are fixed at $m=3, \tau=3$. 
[Fig. 2(c)] especially along the lines of identity (known as LOI, i.e. the middle diagonal line) where perfect continuous line is expected) and in the LMT [Fig. 2(e)] despite the reduction of thick lines (marked with red rectangles). Although the thick lines in the threshold based RP result in clearer diagonal lines and, hence, a higher DET value; this is, however, not necessarily correct, because these thick lines (and block structures) artificially introduce more diagonal and vertical lines; therefore, artificially increasing LAM (i.e., in our example by 0.35). Despite the thick lines in threshold based $\mathrm{RP}$ result in the visually clearer diagonal lines, this is not necessarily correct as they artificially introduce more diagonal lines and block structures that increase vertical lines and hence artificially increasing LAM (i.e. in our example and increase by 0.35 ). This is a problem because such laminarity characteristics are not expected for the Lorenz system. The increase of LAM is also noticed in the LMT approach (by
0.11). Meanwhile, the LM RP [Fig. 2(d)] is dominated by visible long vertical lines which, therefore, drastically increase the LAM when compared to the RP of the Lorenz without noise (increase by 0.69).

To tackle the problems with noisy continuous signals (blocks and vertically extended structures), we propose an additional constraining parameter to the LMT in order to reduce the mentioned artifacts (i.e., block cluster and increase in LAM) and we call this method local minima based RP with 2 parameters (LM2P). This LM2P approach is further explained in Sec. II. Although LM2P is found to improve the reliability of RP and RQA as evaluated from its deviation from the RP constructed from uncontaminated signal (i.e., without noise), the disrupted or discontinuous diagonal lines due to noise are, however, still a bottleneck in deriving a system representative DET value, e.g., such that the deterministic Lorenz would still yield a very low DET value

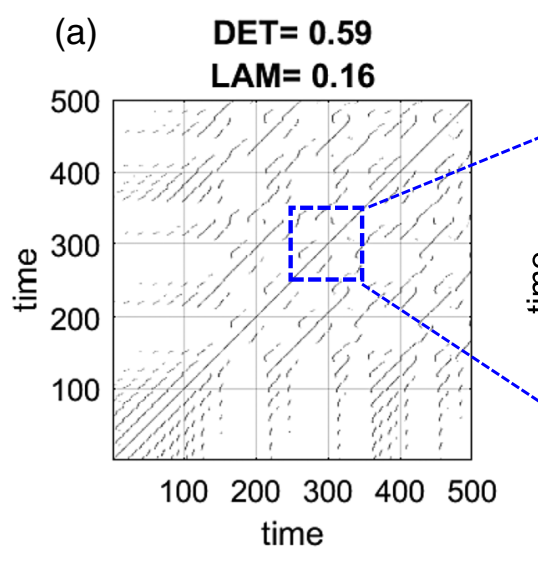

(b)

DET $=0.59$

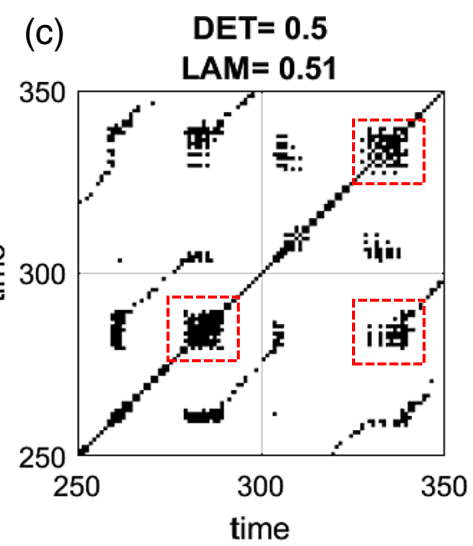

(e)

$\mathrm{DET}=\mathbf{0 . 2}$

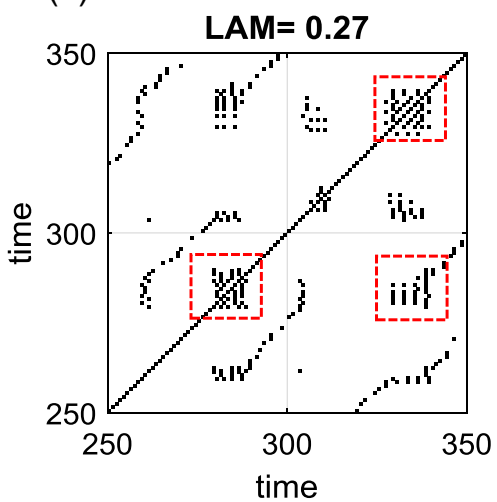

(d)

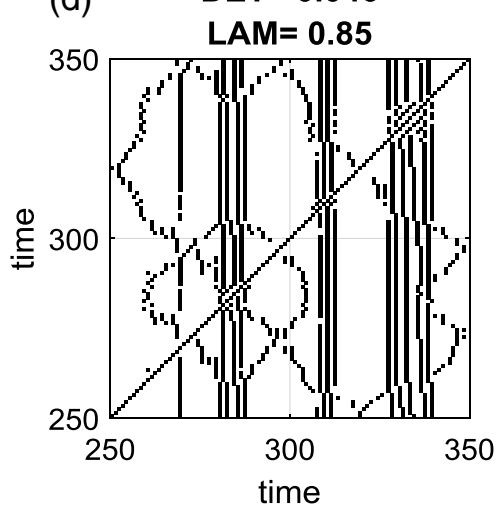

(f)

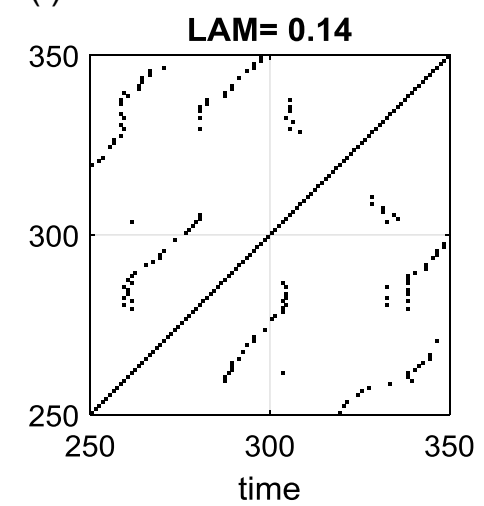

FIG. 2. RPs of the Lorenz series $\left(\frac{d x}{d t}\right.$, with $\sigma=10$, $\rho=28, \beta=\frac{8}{3}$ ) and additionally induced with noise (SNR = $20 \mathrm{~dB}$ ). The following RPs are constructed using (a) LMT of Lorenz with no noise, while (b) is the zoomed-in view of the same RP, (c) distance threshold of $\varepsilon=5$ th percentile of phase distances, (d) LM only, (e) LMT with the same $\varepsilon$ parameter used in (a), and (f) LM2P with the same $\varepsilon$ and $\tau_{m}=10$. The red rectangles in (c) and (e) are used to indicate the mentioned block clusters. The embedding parameters of all RP are fixed at $m=3, \tau=3$. 
(a) $5^{\text {th }}-95^{\text {th }}$ percentile of $\tau_{m}$ and its median:
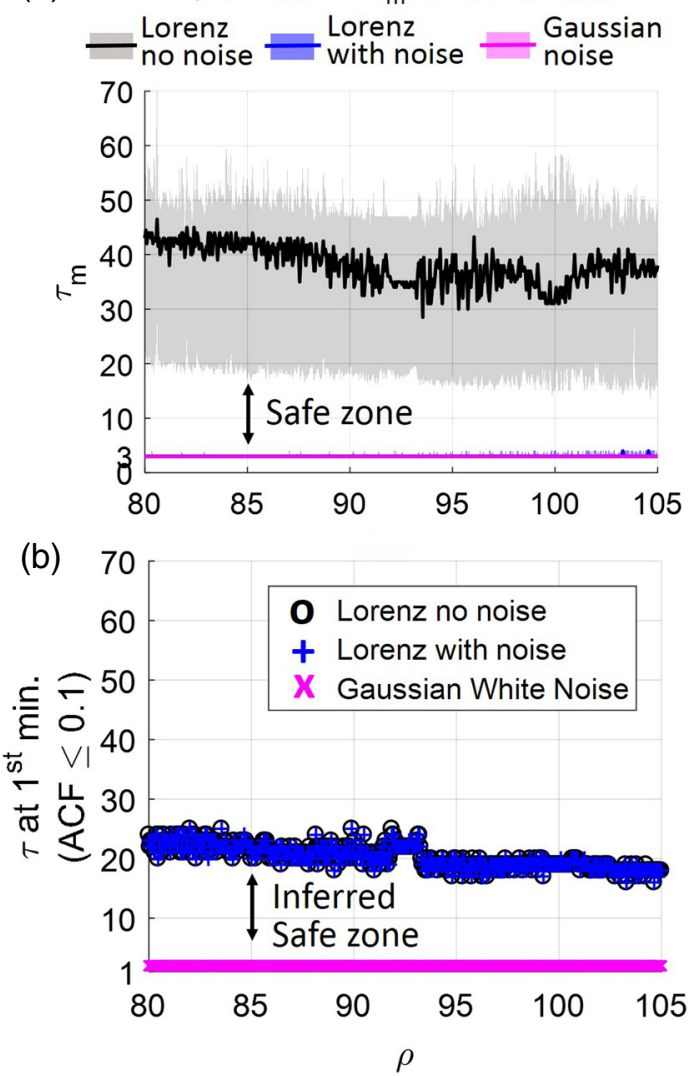

FIG. 3. Safe choice of parameter $\tau_{m}$ by using ACF: (a) Local minima of the phase space distance for Lorenz and Gaussian noise, while (b) is the inferred safe $\tau_{m}$ to choose based on first minimum $\tau$ of auto-correlation (chosen threshold, $A C F \leq 0.1)$.

despite its RP deterministic pattern (i.e., DET of 0.24 instead of 0.59 found in the RP of Lorenz without noise). Such is also noticed in different Lorenz systems with varying $\rho$ parameters (Fig. 3), where DET of the noise induced signals (both LMT and LM2P) show a very low DET value closer to the stochastic system rather than the reference DET generated from the RP of Lorenz with no noise. This reference RP (shown in all figures as Lorenz no noise) is generated using the LM2P approach, although there is hardly any difference with the one generated using the LMT approach (i.e., when no noise is present). Therefore, we also propose a new measure to account for this mentioned diagonally clustered points that do not necessarily form strict diagonal lines as required for the conventional RQA calculation of, e.g., DET. The methods and findings are presented and discussed in Secs. II and III.

\section{LOCAL MINIMA BASED RP WITH 2 PARAMETERS (LM2P)}

The vertical distance between recurrence points in the RP corresponds to the time between recurring states (recurrence time). However, noise can reduce these recurrence times by introducing many new local minima in the distance matrix, and by this artificially adding many new recurrences (visible as clusters and vertical structures in Fig. 2). In order to reduce the number of these noise-induced minima, we introduce a minimum distance $\tau_{m}$ required between the local minima as a new additional parameter. This $\tau_{m}$ in addition to $\varepsilon$ is also illustrated in Fig. 2(d). Because the local minima approach now consists of two parameters (i.e., $\varepsilon$ and $\tau_{m}$ ), we call this approach "local minima with 2 parameters" (LM2P). By doing so, we exclude the minima that are found next to each other due to the induced noise. The resulted minima with reasonable $\tau_{m}$ are expected to separate the potentially recurring pattern despite the local noise. This selection of $\tau_{m}$, however, should be minimized so that one does not exclude the recurring pattern due to large $\tau_{m}$ that potentially overlaps the sequence of the signal. It should also be reasonably sized to avoid the noise artifact. The optimal $\tau_{m}$ as implied by Fig. 3(a) has to be larger than the maximum of the local minima of the phase space distances as found in a random system (i.e., Gaussian white noise) and smaller than the one of the assessed deterministic signal (in this case Lorenz). Such a criterion can be found by using the auto-correlation function (ACF) of the assessed signal. This should be chosen larger than the first minimum lag of white noise, and lower than the first minimum lag of the assessed signal. In this case, we obtained the first minimum by using a threshold of autocorrelation value less than or equal to 0.1 , and we infer the safe choice of $\tau_{m}$ to be below 15 at Lorenz with $\rho=80$ [see Fig. 3(b)]. Since the $\tau_{m}$ of Gaussian noise is found to be around 3, we could infer the safe $\tau_{m}$ for our noisy Lorenz signal to be above 3 (let us say 5) [see Fig. 3(a)] and below 15 as guided by ACF. The RP based on the LM2P approach solves the mentioned issues on thick lines and block patterns as well as the vertical artifacts due to noise. First, for the noisy signal, the LM2P RP [Fig. 2(f)] yields a closer pattern to the one of the uncontaminated time series (i.e., without noise) as seen in Figs. 2(a) and 2(b). Furthermore, the block clusters are now removed and vertical lines due to noise disappear [Fig. 2(f)]. This is confirmed by the low LAM value that is similar to the one of the signal with no noise (difference only 0.02). However, disrupted lines due to noise are still visible to cause low diagonal line based RQA measures (e.g., DET, difference 0.35) despite the agreement of the RP patterns. The conventional RQA measures based on strict diagonal structures (i.e., connected points with $45^{\circ}$ slope) are not always satisfactory especially in the case of noisy signals, where diagonal lines can be disrupted by noise. The disruption of line is prominent and shown to vary with the noise level (see Fig. 4). For instance, noise impact with SNR of $30 \mathrm{~dB}$ already causes the median values (probability exceedance of 0.5 ) of the line length to 1 (i.e., no connected points) and that the relatively longest diagonal line within the RP already drops to $30 \%$. Despite the solution in increasing $\varepsilon$ could be promising in connecting the disrupted and deviated lines, ${ }^{10}$ such an approach undesirably thickens the structures in the RP and eventually causes an artificial increase in diagonal and vertical lines, and, therefore, biases RQA.

\section{DETERMINISM INDICATOR USING DIAGONAL SLIDING WINDOW}

In order to resolve the aforementioned reliability issues (i.e., DET sensitivity to disrupted and deviated diagonal lines), we propose another approach to derive a more robust determinism indicator that is less sensitive to noise. This 
(a)

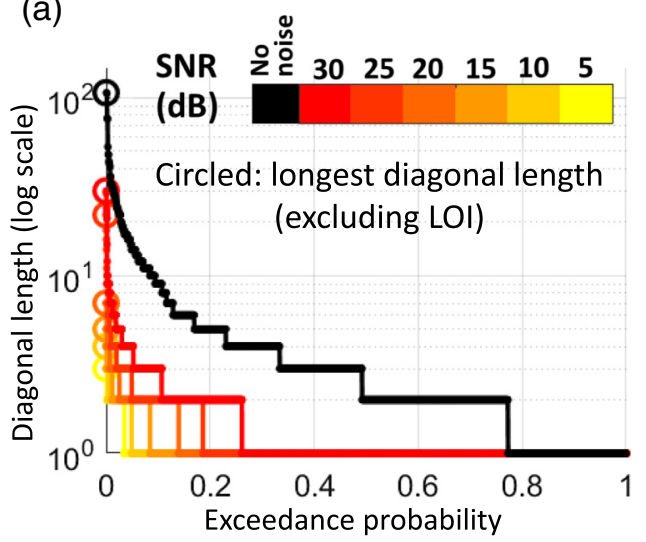

(b)

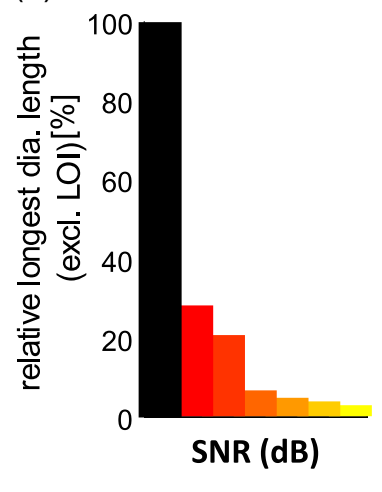

FIG. 4. (a) Probability exceedance of diagonal line lengths and (b) relative longest diagonal line length $(\%)$ of Lorenz of $\rho=80$. with response to induced noise, i.e., indicated by signal to noise ratio (SNR). approach uses a sliding window $\left(w_{i}\right)$ of size $w s$ and iterates at a lag of $\tau_{w}$ (distance from the main diagonal LOI) from the left corner of the RP until the end point of the RP (right corner) minus the window size $(N-w s)$. The conceptual diagram of this method is presented in Fig. 5(a). The RP of a deterministic signal tends to form a cluster of diagonal structures despite the wiggly and disrupted lines (i.e., up to the user tolerance deviation criteria set by $w s$, and maximum gap or discontinuity distance of $D(\max )$ ) as exemplified in the rotated view of the window for the sub-figure [Fig. 5(b)] RP of Lorenz without noise, (c) Lorenz with noise using LM2P, (d) Lorenz with noise using LMT, and (e) RP of a random signal (i.e., Gaussian noise) with typical scattered recurrence points all over the space. (a)

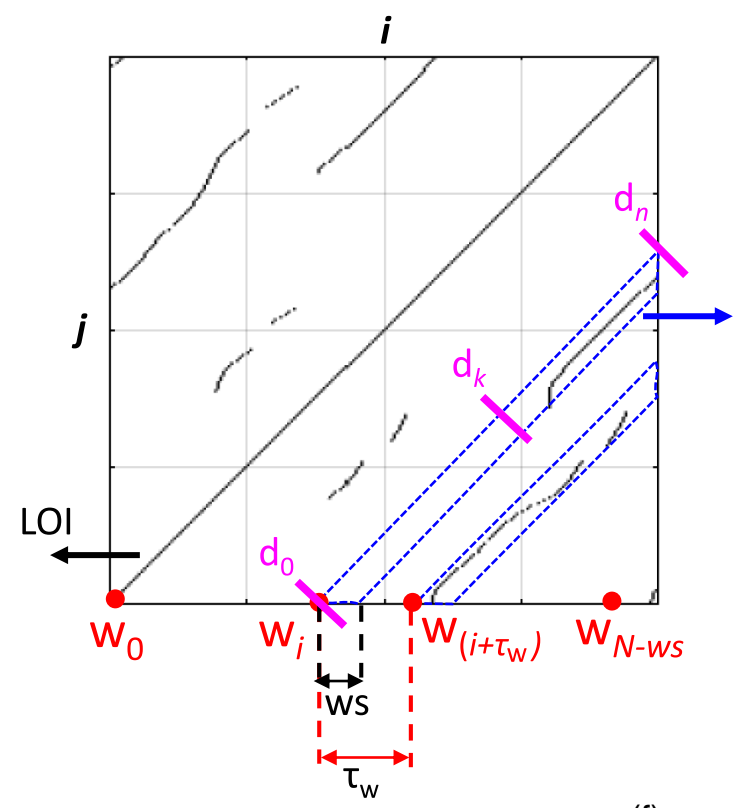

(f)

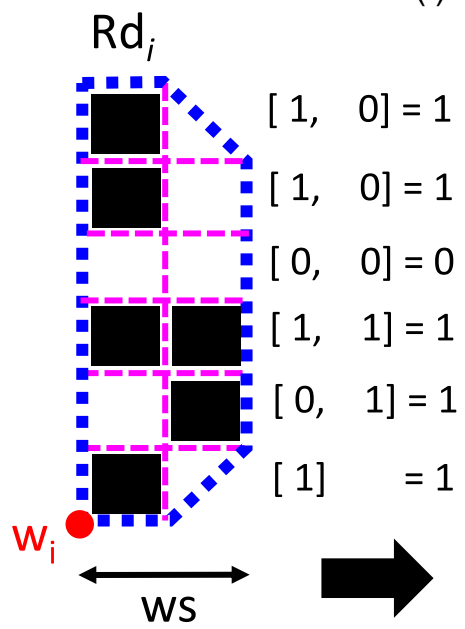

(b)

(c)

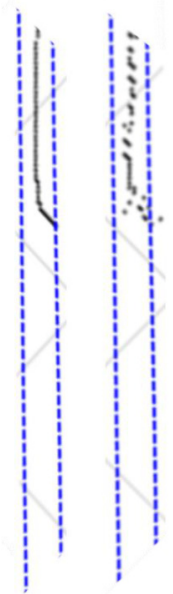

(d)

(e)

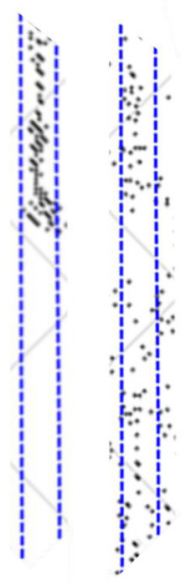

FIG. 5. Concept of the diagonal sliding window: (a) A considerably small $w s$ size window $\left(w_{i}\right)$ slides diagonally through the RP. For each window, $R R_{w_{i}}$ and $D D E T_{w_{i}}$ are calculated [Eqs. (5) and (6)]. Subfigures (b) to (e) show a zoom in the rotated view of a diagonal window to exemplify an RP of Lorenz without noise, RPs with noises constructed with LMT and LM2P, and an RP of Gaussian white noise, respectively. Sub-figure (f) with reference to (a) shows the calculation concept of the diagonal distance between ( $D$ or $\Delta k$ ) recurring points at each window (rotated view) derived from an aggregate of cells (OR logic) from $i$ to $i+w s-1$. 
For each $w_{i}$ the recurrence rate of the window $\left(R R_{w_{i}}\right)$ is calculated as the ratio of the number of recurrence points $\left(R_{w_{i}}\right)$ over the area of the window $A_{w_{i}}$ [Eq. (5)]. In this approach, $w s$ should be set considerably small ( 2 or 3 ) to consider only small wiggling or deviation of the diagonal lines due to noise. Such a limited window, e.g., with $w s=2$, allows each perpendicular cell or point (i.e., $R_{d_{i}}$ binary values) along the length of the window $(i, \ldots, i+w s-1)$ to be aggregated like a logical OR function as $d_{k}$. In addition, for every $d_{k}$ at window $w_{i}$, the diagonal recurrence point distance along the axis $k$ is calculated between each consecutive recurrence points as $D$ or $\Delta k$ [Fig. 5(f)]. The ratio of frequency distribution of the gap distance that is within the threshold $D(\max )$ is the emphasis for our calculation. In this case, the user should allow minimal gap tolerance to consider such discontinuity as the diagonal line (e.g., gap of 1 or 2 point distance). These variables are then used to formulate an alternative determinism indicator $D D_{w_{i}}$ for each window $\left(w_{i}\right)$ and $D D E T$ as the index

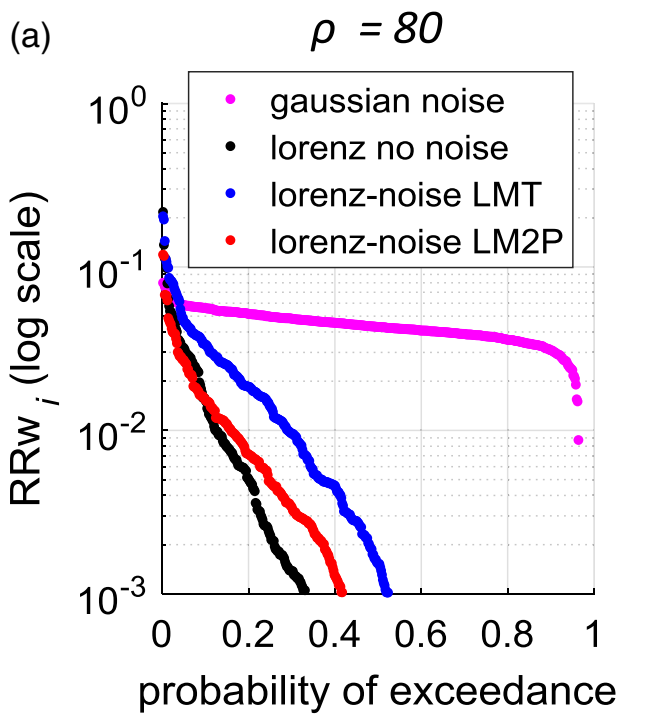

for the whole RP:

$$
R R_{w_{i}}=\frac{1}{A_{w_{i}}} \sum_{i, j=w_{i}}^{n} R_{w_{i, j}},
$$

where $A_{w_{i}}$ is the area of the diagonal window (i.e., number of cells in each window) and $R_{w_{i, j}}$ is the recurrence point within the window calculated from the starting point of the window $\left(w_{i}\right)$ up to the maximum possible length $(N-w s)$.

$$
\begin{aligned}
& D D E T=\log \left(\frac{\frac{1}{N} \sum_{i}^{N} D D_{w_{i}}}{\frac{1}{N} \sum_{i}^{N} R R_{w_{i}}}\right), \\
& D D_{w_{i}}=\frac{\sum_{j}^{D(\max )} D_{j} P\left(D_{j}\right)}{\sum_{j}^{N} l_{w_{i}}-D_{j} P\left(D_{j}\right)}, \\
& D=\Delta k, \text { for }\left\{d_{k} \in \mathbb{B}: d_{k}=1\right\}, \\
& d_{k}=\vee_{i}^{n} R_{d_{i}}, \quad n=i+w s-1,
\end{aligned}
$$

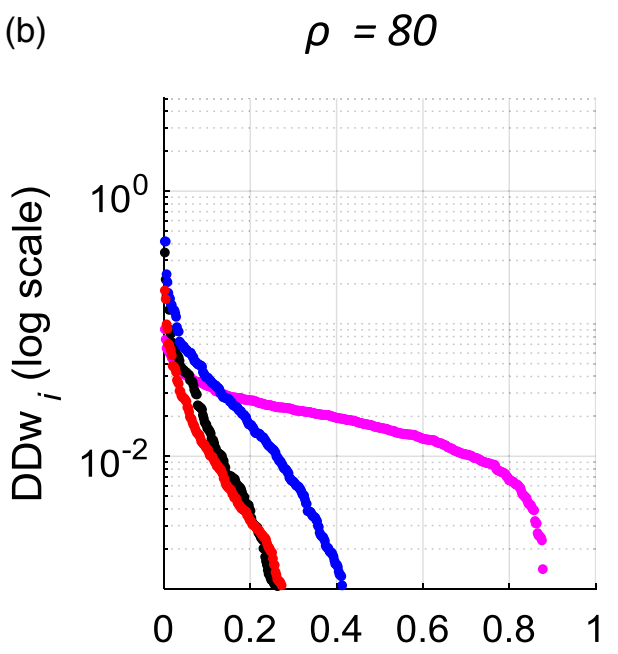

probability of exceedance
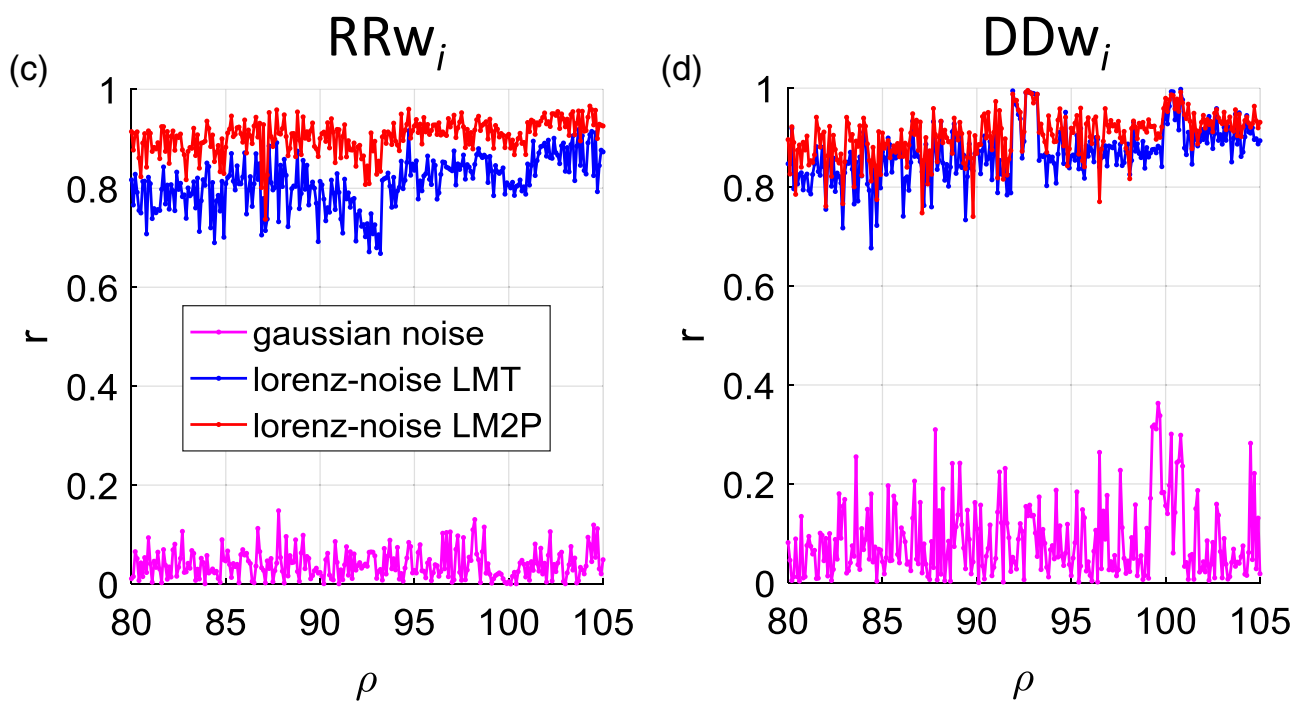

FIG. 6. Probability of exceedance of (a) $R R_{w_{i}}$ and (b) $D D_{w_{i}}$ values of diagonal windows between Lorenz no noise and the LMT and LM2P of the one with noise (shown here is only Lorenz with $\rho=80$ ), and Gaussian noise. Correlation coefficient $r$ of (c) $R R_{w}$ and (d) $D D E T$ for all RPs with reference to the RP of Lorenz with no noise. The embedding parameters of all the assessed RPs are fixed at $m=3, \tau=10, \varepsilon=5$ th, $\tau_{m}=10 w s=2$, and $\tau_{w}=2$. 
where $d_{k}$ is calculated as the logical OR aggregate function of horizontal cells $R_{d_{i}}$ within the window size ws (from $R_{d_{i . . n}}$, where $\left.n=i+w s-1\right) . P\left(D_{j}\right), j=\left\{1, \ldots, N_{D}\right\}$ is the frequency distribution of the gap distance $k$ between consecutive diagonal recurrence points $\left(d_{k} \equiv 1\right)$, where $D(\max )$ is the maximum threshold of distance allowed, $l_{w i}$ is the maximum length of $w_{i}\left(l_{w_{i}}=n_{k}\right)$, and $N_{D}$ is the absolute number of those distances stored in $D$.

The calculated $R R_{w_{i}}$ and $D D_{w_{i}}$ of every window are then compared for their probability of exceedance. Based on Lorenz with parameter $\rho=80$, the distributions of the exceedance are distinct between those of Lorenz and Gaussian processes with better performance of LM2P over LMT (i.e., closer to the one of Lorenz with no noise) especially for $D D_{w_{i}}$ [Figs. 6(a) and 6(b)]. Furthermore, the correlation coefficient ( $r$ ) of $R R_{w_{i}}$ and $D D_{w_{i}}$ between the RP of the Lorenz signal with no noise as reference and the other RPs are assessed, respectively, for varying $\rho$ [Figs. 6(c) and 6(d)]. Unlike the conventional RQA (i.e., DET is low for the noisy Lorenz signal and appears closer to the one of Gaussian noise and thus, the correlation to the reference is low), the correlation between LM2P RP of the reference (i.e., no noise) and the noisy signal is higher.

Furthermore, the resulted DDETs for varying $\rho$ are also plotted in comparison with the DET values of Lorenz with no noise as reference [Fig. 7(b)]. Although the DDET values of Lorenz with noise (both LMT and LM2P) are slightly lower than the one without noise, their values are rather distinctive from the one of Gaussian noise. Meanwhile, two periodic windows ( $P 1$ and $P 2)$ of the Lorenz system as indicated by the maximum Lyapunov exponent below 0 [Fig. 7(a) (red)] are also captured by the DDET measure, while the RQA DET could only capture the high deterministic features of Lorenz in the 2nd periodic window (P2). DDET values can be seen to range from approximately 2 to 3 for the periodic system, fluctuating around a value of 1 for the chaotic regime, and dropped to almost -1 when it is Gaussian noise or the stochastic process.

Although a higher embedding dimension could further insinuate the implicit deterministic features and, hence, increase DET values, the impact of induced noise as shown in Fig. 8 would still result in distinct difference (i.e., underestimation) as compared to the signal without noise. In contrast, DDET values of the noisy Lorenz are found to be much closer to the Lorenz without noise regardless of the embedding dimension. This differentiates stochastic systems like white noise further from the deterministic signal. Furthermore, applying LM2P in this case does not require to choose high embedding. The LM2P approach outperforms the other approach for all $\rho$ parameters [Fig. 7(b)] and with varying embedding dimensions (Fig. 8).

Despite the selection of optimum $w s$ being attributed to the amount of noise, i.e., the lower the signal to noise ratio (SNR), the expected deviation is larger, and the large choice of $w s$ could result in the recurring sequence being aggregated within a window. In our test case, we focus on the example of

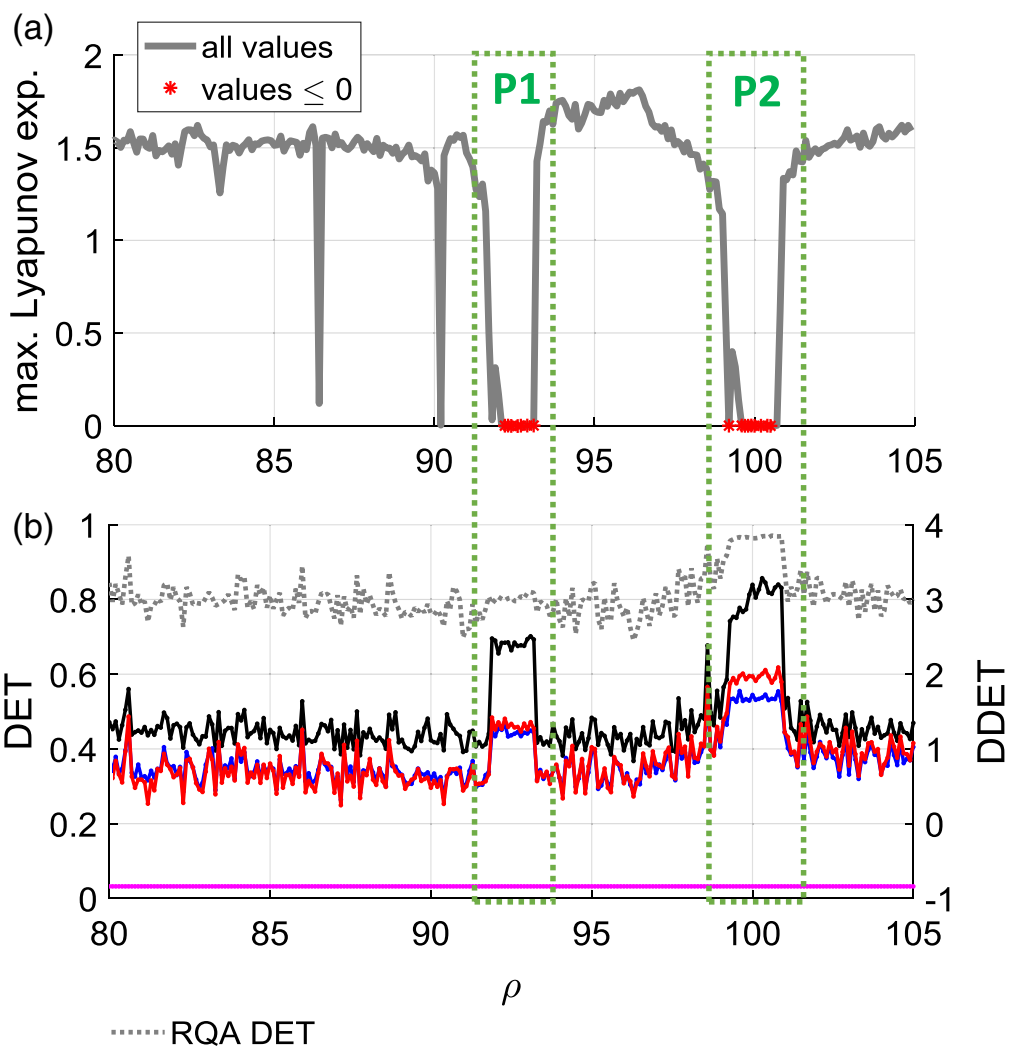

DDET values for:
FIG. 7. (a) Maximum Lyapunov exponent of the Lorenz system with varying $\rho$ parameters (with values below 0 indicating periodic dynamics highlighted in red). (b) DDET values of Gaussian noise and Lorenz with varying parameters with reference to DET. Green boxes emphasize the two Lorenz periodic windows $\mathrm{P} 1$ and $\mathrm{P} 2$ (indicated by the max. Lyapunov exponent below 0$)$. The RP measures are calculated based on the embedded approach: $m=3, \tau=10, \varepsilon=5$ th, and $\tau_{m}=10$ (for LM2P), and $w s=2$ and $\tau_{w}=2$ (for the DDET calculation). 
(a)

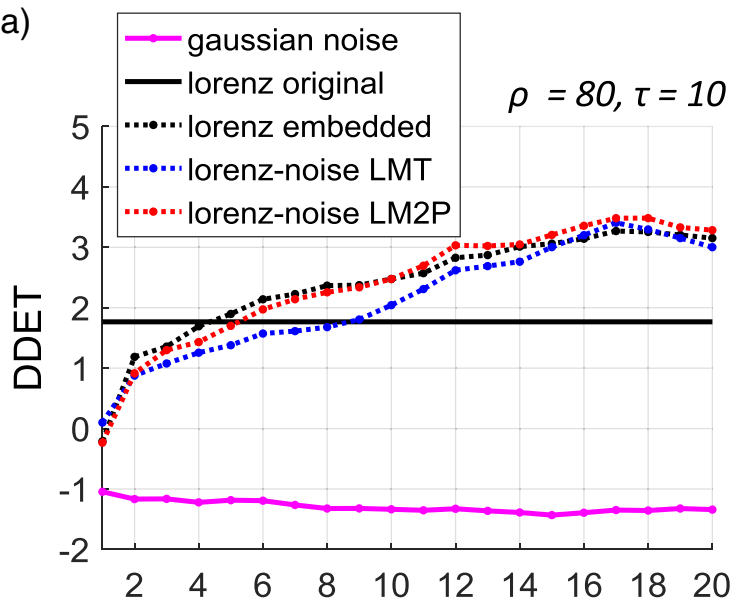

(b)

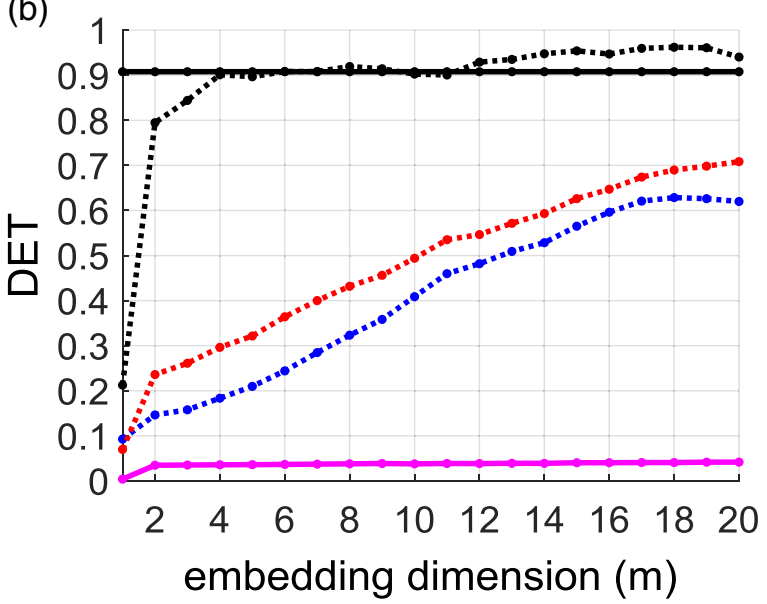

FIG. 8. (a) Impact of the embedding parameter ( $m$ on the DDET measure). Shown here is the example of $w s$ for the full Lorenz system (i.e., created without embedding, using 3 variables) RP (LM2P) set for $2 \leq w s \leq 10$ and additionally $w s=50$ and Gaussian noise with $w s=2$ as the reference.

the Lorenz series with $20 \mathrm{~dB}$ SNR, which is already considered to be quite a prominent noise contamination case. With this SNR, the choice of $w s=2$ is already shown to be effective to derive a representative DDET (i.e., close to the signal

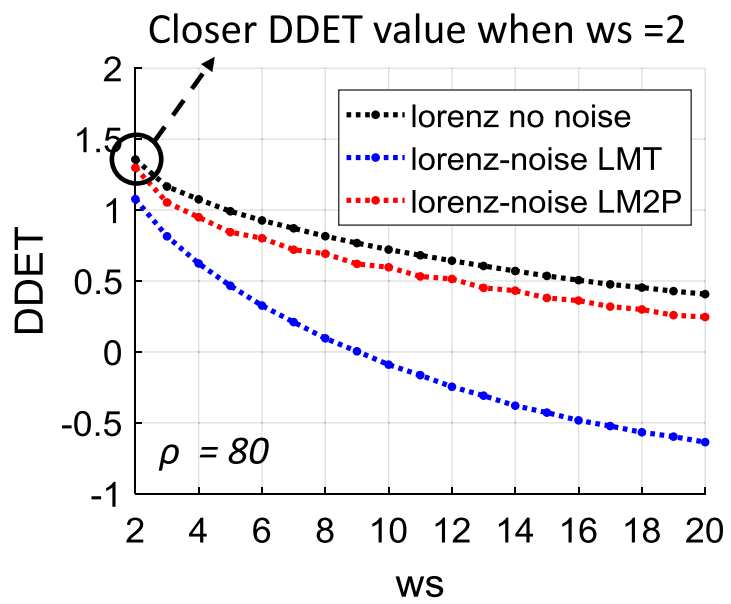

FIG. 9. (a) Impact of the window size parameter ( $w s$ on the DDET measure). Shown here is the example for DDET of the embedded Lorenz system using the LMT and LM2P approaches in response to changing $w s$ in comparison with Lorenz without noise. (a)
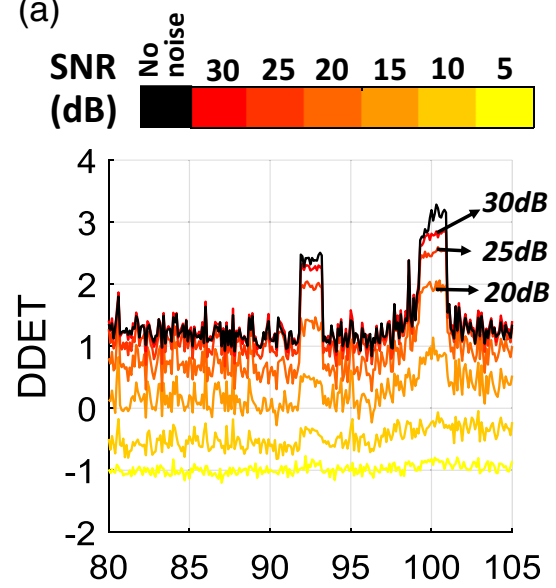

(b)

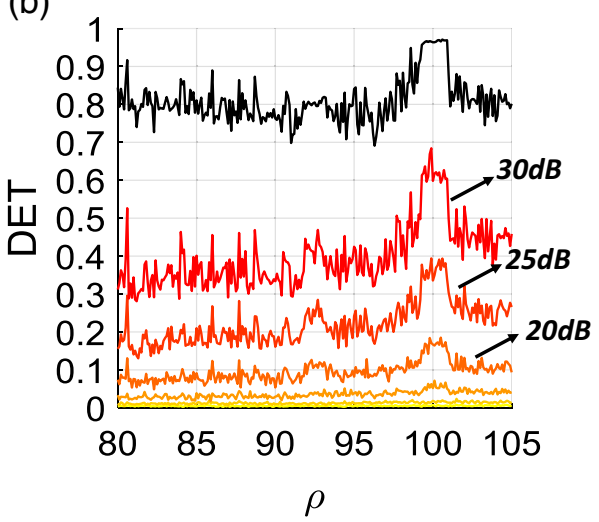

FIG. 10. (a) Impact of the induced noise SNR level on the DDET and DET measures. Shown here is the example of the noisy Lorenz series with SNR from 5 to $30 \mathrm{db}$ as compared to Lorenz with no noise. All measures are based on RPs generated using LM2P and the embedded approach with $m=3$, $\tau=10, \varepsilon=5$ th, $\tau_{m}=10 w s=2$, and $\tau_{w}=2$.

without noise), and as shown in Fig. 9, the increase of ws would only decrease the reliability of DDET (i.e., DDET values between non-noisy and noisy signals start to distant apart and that the DDET values of the deterministic signal start to decrease). In this figure, it is also shown that the LM2P approach would yield more robust DDET values with respect to the increase of $w s$ in contrast to the LMT approach where the decrease of DDET deviates further from the non-noisy signal.

Moreover, this example can be used to study the sensitivity of the new approach with respect to noise. The DDET values for SNR ranging from 30 to $20 \mathrm{~dB}$ are still close to the DDET values of the non-noisy signal (Fig. 10). On the contrary, DET is very sensitive to noise, and the drop in SNR to $20 \mathrm{~dB}$ causes a drastic change in the DET and implies a closer relationship to a stochastic process. However, in the case of an extremely noisy signal, i.e., SNR below $20 \mathrm{~dB}$, even DDET cannot be longer deemed to be reliable.

\section{CONCLUSION}

(1) RP artifacts such as thick lines can be reduced using the LMT (i.e., introduced by Schultz et al. ${ }^{8}$ ) and the LM2P methods (i.e., introduced in this paper). However, when noise is present in the continuous system, the LMT approach is 
still shown to contain block like clusters and artificial vertical lines that eventually increase the RQA measure LAM. This, however, can be resolved by using the LM2P approach, where an additional parameter constraint (i.e., minimum distance $\tau_{m}$ between the local minima) is introduced in addition to the threshold. The parameter $\tau_{m}$ can be guided by using the location of the first zero auto-correlation function (ACF). The recommended $\tau_{m}$ should be smaller than the first zero ACF but larger than the ACF of the stochastic process.

(2) Although the LM2P (and LMT) approach reduces the mentioned artifacts and visually appears to have agreement between RPs created from pure and noisy signals (i.e., a closer representation of LAM values), their conventional RQA calculated based on diagonal line lengths (i.e., DET) is heavily underestimated. This is due to the impact of the induced noise that disrupts and deviates the recurrence points. Therefore, the strict quantification based on connected points that form $45^{\circ}$ diagonal lines would fail to capture the recurring dynamics' property.

(3) To resolve the RQA reliability with mentioned disrupted and deviated diagonal lines, an alternative approach for calculating a determinism indicator is proposed. This uses a diagonal sliding window concept with a minimal window size $(w s)$ designed to capture the mentioned deviated lines at each window time lag $\left(\tau_{w}\right)$.

(4) To account for the disrupted lines (i.e., cluster of points with minimal distance), we propose an index measured from the distribution of this minimal diagonal point distance, and their recurrence rate at each window iteration $\left(w_{i}\right)$. This allows disrupted points with minimum distance to be considered as a diagonal line and, hence, provides a DET equivalent measure.

(5) The new measure is able to capture the deterministic property of the Lorenz system (for varying parameter $\rho$ from 80 to 110) despite the induced noise with signal to noise ratio of $20 \mathrm{~dB}$, as assessed from their window's $R R_{w}$ and $D D_{w_{i}}$ correlation agreement, distribution, and $D D E T$ variation for different $\rho$ values as compared to the system without noise. In addition, it also captures the two periodic windows of the Lorenz system in both noisy and non-noisy signals, whereas the conventional RQA-DET measure fails to capture them. The conventional DET measure also tends to distinctly underestimate the determinism and, hence, appears to be closer to the indication of a stochastic process when the induced noise is present. Furthermore, the use of DDET allows minimal embedding dimension to match the noisy system with the nonnoisy one, and their performance is, in general, rather constant in spite of the embedding.
(6) However, it is worth to note that the magnitude range of this measure differs from the conventional DET measure. The challenge remains in setting up a standard of range for which a more intuitive scale of determinism can be easily inferred. Nevertheless, the new DDET measure and the diagonal sliding window are promising concepts when the known continuous system dynamics is induced by noise, which is very common in real world observations. More research could focus on the more specific attribution of the measures and different types of noise. Furthermore, the relation of the signal to noise ratio to the window size (ws) necessary to capture the expected deviation and the maximum diagonal distance to consider the tolerance of line disruption should be elaborated in future studies.

\section{ACKNOWLEDGMENTS}

This research was carried out within the Research Training Group "Natural Hazards and Risks in a Changing World" (NatRiskChange; Grant No. GRK 2043/1) funded by the Deutsche Forschungsgemeinschaft (DFG). In addition, this work has been financially supported by the German Research Foundation (DFG Project Nos. MA 4759/9-1 and MA4759/8) and the European Unions Horizon 2020 Research and Innovation programme under the Marie 499 Skłodowska-Curie Grant Agreement No. 691037 (project QUEST).

${ }^{1}$ N. H. Packard, J. P. Crutchfield, J. D. Farmer, and R. S. Shaw, "Geometry from a time series," Phys. Rev. Lett. 45, 712-716 (1980).

${ }^{2}$ F. Takens, "Detecting strange attractors in turbulence," in Dynamical Systems and Turbulence, Warwick 1980, Lecture Notes in Mathematics Vol. 898 (Springer-Verlag, 1981), pp. 366-381.

${ }^{3}$ J.-P. Eckmann, S. O. Kamphorst, and D. Ruelle, "Recurrence plots of dynamical systems," Europhys. Lett. 4, 973 (1987).

${ }^{4}$ N. Marwan, M. Carmen Romano, M. Thiel, and J. Kurths, "Recurrence plots for the analysis of complex systems," Phys. Rep. 438, 237-329 (2007).

${ }^{5}$ J. P. Zbilut and C. L. Webber, "Embeddings and delays as derived from quantification of recurrence plots," Phys. Lett. A 171, 199-203 (1992).

${ }^{6}$ L. L. Trulla, A. Giuliani, J. P. Zbilut, and C. L. Webber, "Recurrence quantification analysis of the logistic equation with transients," Phys. Lett. A: Gen. At. Solid State Phys. 223, 255-260 (1996).

${ }^{7}$ N. Marwan, "How to avoid potential pitfalls in recurrence plot based data analysis," Int. J. Bifurcat. Chaos 21, 1003-1017 (2010). arXiv: 1007.2215.

${ }^{8}$ A. P. Schultz, Y. Zou, N. Marwan, and M. T. Turvey, "Local minima-based recurrence plots for continuous dynamical systems," Int. J. Bifurcat. Chaos 21, 1065-1075 (2011).

${ }^{9}$ D. Wendi, N. Marwan, and B. Merz, "In search of determinism-sensitive region to avoid artefacts in recurrence plots," Int. J. Bifurcat. Chaos 28, 1850007 (2018).

${ }^{10}$ M. Thiel, M. C. Romano, J. Kurths, R. Meucci, E. Allaria, and F. T. Arecchi, "Influence of observational noise on the recurrence quantification analysis," Physica D 171, 138-152 (2002). 